\title{
Emission of Fast Non-Maxwellian Hydrogen Atoms in Low-Density Laboratory Plasma
}

\author{
Christian Brandt ${ }^{1,2, a)}$, Oleksandr Marchuk ${ }^{2}$, Albrecht Pospieszczyk ${ }^{2}$ and Sven \\ Dickheuer $^{2}$ \\ ${ }^{1}$ Max-Planck-Institute for Plasma Physics, Wendelsteinstr. 1, 17491 Greifswald, Germany \\ ${ }^{2}$ Forschungszentrum Jülich GmbH, Institut für Energie- und Klimaforschung - Plasmaphysik, 52425 Jülich, \\ Germany
}

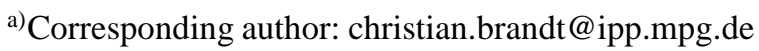

\begin{abstract}
The source of strong and broad emission of the Balmer- $\alpha$ line in mixed plasmas of hydrogen (or deuterium) and noble gases in front of metallic surfaces is a subject of controversial discussion of many plasma types. In this work the excitation source of the Balmer lines is investigated by means of optical emission spectroscopy in the plasma device PSI-2. Neutral fast non-Maxwellian hydrogen atoms are produced by acceleration of hydrogen ions towards an electrode immersed into the plasma. By variation of the electrode potential the energy of ions and in turn of reflected fast atoms can be varied in the range of $40-300 \mathrm{eV}$. The fast atoms in front of the electrode are observed simultaneously by an Echelle spectrometer $(0.001 \mathrm{~nm} /$ channel $)$ and by an imaging spectrometer $(0.01 \mathrm{~nm} / \mathrm{channel})$ up to few $\mathrm{cm}$ in the plasma. Intense excitation channels of the Balmer lines are observed when hydrogen is mixed with argon or with krypton. Especially in Ar-H and Ar-D mixed plasmas the emission of fast hydrogen atoms is very strong. Intermixing hydrogen with other noble gases ( $\mathrm{He}, \mathrm{Ne}$ or $\mathrm{Xe}$ ) one observes the same effect however the emission is one order of magnitude less compared to $\mathrm{Kr}-\mathrm{H}$ or $\mathrm{Kr}-\mathrm{D}$ plasmas. It is shown, that the key process, impacting this emission, is the binary collision between the fast neutral hydrogen atom and the noble gas atom. Two possible sources of excitation are discussed in details: one is the excitation of hydrogen atoms by argon atoms in the ground state and the second one is the process of the so-called excitation transfer between the metastable states of noble gases and hydrogen. In the latter case the atomic data for excitation of Balmer lines are still not available in literature. Further experimental investigations are required to conclude on the source process of fast atom emission.
\end{abstract}

Keywords: Balmer lines, excitation transfer, non-Maxwellian atoms

PACS: $25.40 . \mathrm{Kv}, 32.80 . \mathrm{Xx}, 52.20 . \mathrm{Hv}, 52.25 . \mathrm{Ya}$

\section{INTRODUCTION}

The significantly broadened Balmer or Lyman series emitted by hydrogen or deuterium atoms is a highly debated phenomenon observed in the atmosphere of stellar and planetary plasmas [1, 2], low temperature plasmas of hollow cathodes [3], radio frequency induction plasmas [4] or microplasma jets at atmospheric pressures [5]. In many cases the presence of noble gases stimulates the emission of hydrogen lines, i.e., either the intensity is increased or the lines become extensively broadened. In contrast to the sustained experimental data there is an ongoing controversial discussion on the atomic or molecular processes leading to the observed emission. If in one case the interaction of ions with molecules is considered to be the major emission source of the fast component, the binary interaction (such as charge-exchange or excitation) between the hydrogen atoms and noble gas atoms is considered to be a primary source of broad emission in other cases. One of the most natural mechanism of generation of fast atoms in laboratory plasmas is a result of plasma surface interaction. Walls or negatively charged surfaces generate sheath regions with electric fields being extended in the order of a few Debye lengths [6]. The electrostatic potential $\phi$ in the sheath changes from the plasma potential $\phi_{p}$ in the bulk plasma to the wall potential $\phi_{\text {wall }}$. In the electric field of the sheath positively charged ions are accelerated towards the wall and gain kinetic energy. At the wall their kinetic energy is given by $E_{k i n} \sim Z e_{0} \phi_{\text {sheath }}$ with $Z$ being charge state of the ion, $e_{0}$ the electron elementary charge and $\phi_{\text {sheath }}=\phi_{\text {wall }}-\phi_{p}$. A considerable fraction of the incident ions is reflected at the surface as neutrals after exchanging momentum and 
energy with the atoms of the wall [6]. Thus, the atoms with the energy $E_{k}$ exceeding the energies of the Maxwellian ions in the plasma $E_{k} \gg T_{i}$, where $T_{i}$ is the ion temperature, re-enter the plasma. By observing and understanding the emission of these atoms in the plasma one obtains new insights on the development of light and laser sources, ion solid interaction [7] and the development of theoretical gas kinetics models [8].

In this paper we present emission spectra produced by fast $\mathrm{H}$ or fast $\mathrm{D}$ atoms in the energy range $40-300 \mathrm{eV}$ in a linear magnetized plasma. First, a brief discussion of the spectroscopic setup will be given. Next, we show the experimental results of the emission caused by fast atoms and its general characteristics, without discussing the source of the emission. Eventually we present experimental data of mixed plasmas of D with $\mathrm{He}, \mathrm{Ne}, \mathrm{Ar}, \mathrm{Kr}$ and $\mathrm{Xe}$ gas. We prove that the source of emission of fast atoms is a result of binary collisions between noble gases and hydrogen atoms and discuss the possible atomic processes leading to the observed emission.

\section{EXPERIMENTAL SETUP}

The experiments are performed in the linear magnetized plasma device PSI-2 [9]. The experimental device and the spectroscopical setup is shown in Figure 1. A set of six magnetic field coils creates a linear magnetic field axis. Plasma is produced by a hot-cathode reflex-arc discharge using a heated $\mathrm{LaB}_{6}$ cathode. In the homogeneous part of the plasma column an electrode can be radially inserted into the plasma by a mechanically driven manipulator. A target plate of the size $1 \mathrm{~cm} \times 1 \mathrm{~cm}$ is mounted on a actively water-cooled head of the manipulator and an electrostatic potential between $0 \mathrm{~V}$ down to $-300 \mathrm{~V}$ relative to the plasma vessel can be applied to this target in order to accelerate ions. Radial profiles of plasma parameters, such as electron density $n_{e}(r)$ and electron temperature $T_{e}(r)$, are routinely measured using a swept Langmuir probe at the upstream port position in the plasma. In the evaluation of the current voltage characteristics measured in mixed plasmas the average ion mass of gases weighted with the gas flow rates has been used. The vacuum vessel is equipped with several gas inlets, one is located at the source, and three others at the main plasma vessel. Via a gas flow control system several gases, each adjusted to a selected gas flow rate, can be flushed into the vacuum chamber simultaneously. In the present experiments hydrogen or deuterium and noble gases, i.e., helium, neon, argon, krypton and xenon have been used as working gases.

The optical emission of the plasma is measured with two different spectrometers along two different lines-of-

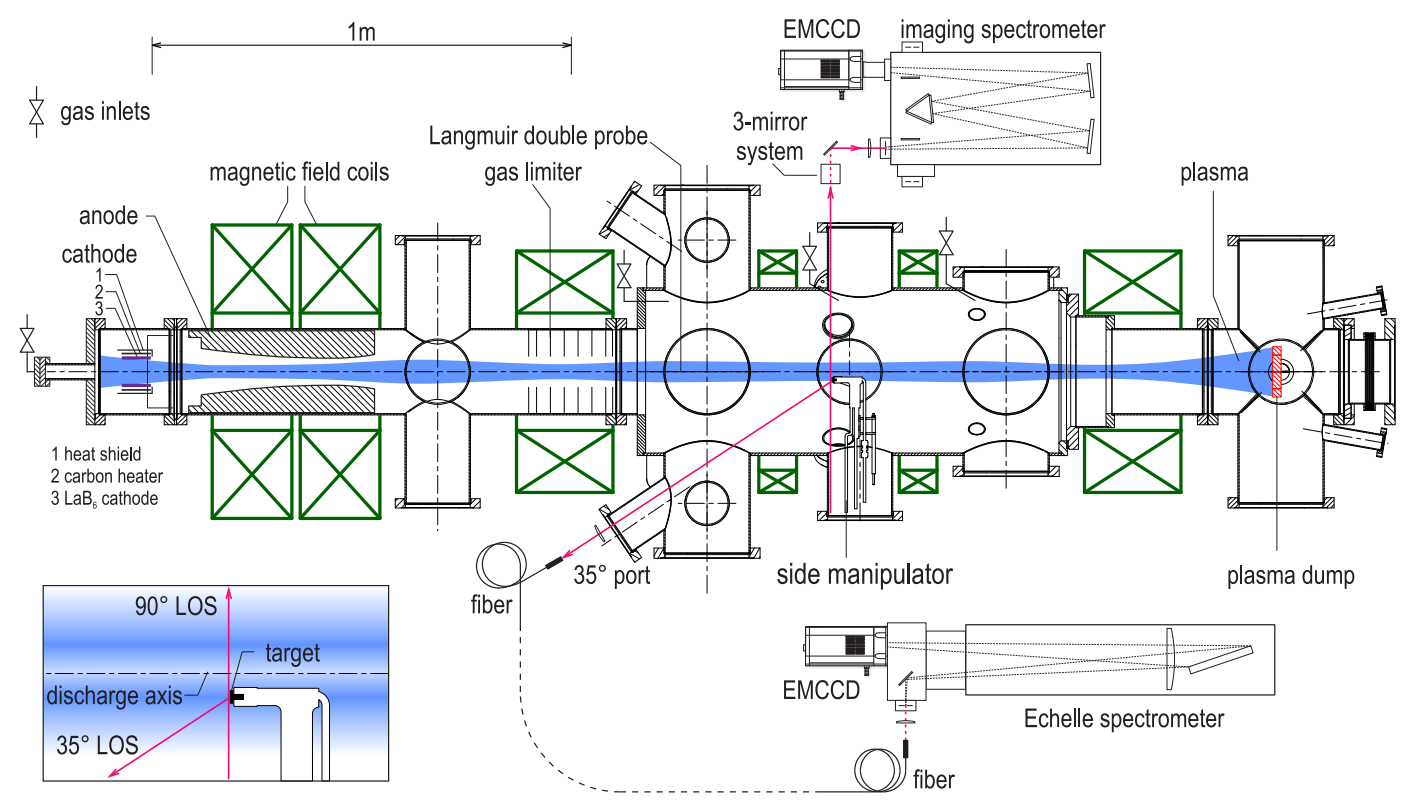

FIGURE 1. (Color online) The linear magnetized plasma device PSI-2 and the spectroscopy setup as used in the present work. The two lines-of-sight (LOS) used for spectroscopy of the plasma in front of the target electrode surface are drawn in magenta color. The blow up shows the head of the radially movable side manipulator and the mounted target electrode. The plasma indicated by the shaded blue in the PSI-2 device has a hollow density profile, i.e., the density has a local minimum on the discharge axis and two maxima. The target center is placed in one maximum. 
sight. Figure 1 shows the two ports used for spectroscopic access. A high-resolution spectrometer equipped with an Echelle grating in high orders $(\approx 40)$ Littrow configuration with a dispersion of about $0.7-1.0 \AA / \mathrm{mm}$, a focal length of $1000 \mathrm{~mm}$, a groove density of 79 grooves $/ \mathrm{mm}$ and blaze angle of $76^{\circ}$ has been used. The entrance slit of the spectrometer is illuminated by two optical fibers observing the plasma using two different lines-of-sight $\left(35^{\circ}\right.$ and $90^{\circ}$ relative to the plasma axis). An $H_{\alpha}$ filter $\left(\Delta \lambda_{F W H M}=1.5 \mathrm{~nm}\right)$ has been placed in front of the entrance slit of the spectrometer. The spectra of two lines-of-sight are imaged at two vertical positions of the exit slit of the spectrometer. An EMCCD camera (ANDOR iXon Ultra 888 [10]) with a chip size 1024 px $\times 1024$ px and pixel size of $13 \mu \mathrm{m}$ is attached to the spectrometer allowing a spectral dispersion of about $0.7-1.0 \mathrm{pm} / \mathrm{px}$ in the horizontal direction of the chip. The lines-of-sight are focused on the target electrode surface having a spot size of approximately $3 \mathrm{~mm}$ in diameter. A second spectrometer, the Princeton Instruments imaging spectrometer ACTON Series SP2750, was used to measure the spatial profile of emitted radiation along the same line-of-sight with $90^{\circ}$ observation angle. The spectrometer has a focal length of $750 \mathrm{~mm}$, a groove density of 1200 grooves $/ \mathrm{mm}$ and a spectral dispersion of $11.0 \mathrm{~A} / \mathrm{mm}$ [11]. The Andor Newton 971 EMCCD detector [12] with a chip size of $1600 \mathrm{px} \times 400 \mathrm{px}$ and pixel size of $16 \mu \mathrm{m}$ is coupled to the exit slit of the spectrometer (1600 px spectral resolution, $400 \mathrm{px}$ spatial resolution). Emission can be measured along radial direction $(r)$ or along axial direction $(z)$ by using a three-mirror system in front of the entrance slit allowing a $90^{\circ}$ rotation of the image of the plasma.

\section{EXPERIMENTAL RESULTS}

Figure 2 summarizes the results of observation for (almost) pure D plasma and Ar-D plasma with and without applied negative potential to the target electrode. In case of $\mathrm{D}$ dominated plasma [Figs. 2(a,c)] no variation in the usual emission of the $\mathrm{D}_{\alpha}$ line is observed by increasing the energy of incident ions to $\approx 110 \mathrm{eV}$ at different lines-of-sight. The line shape remain the same and neither observation at $35^{\circ}$ nor at $90^{\circ}$ angle shows considerable changes or new
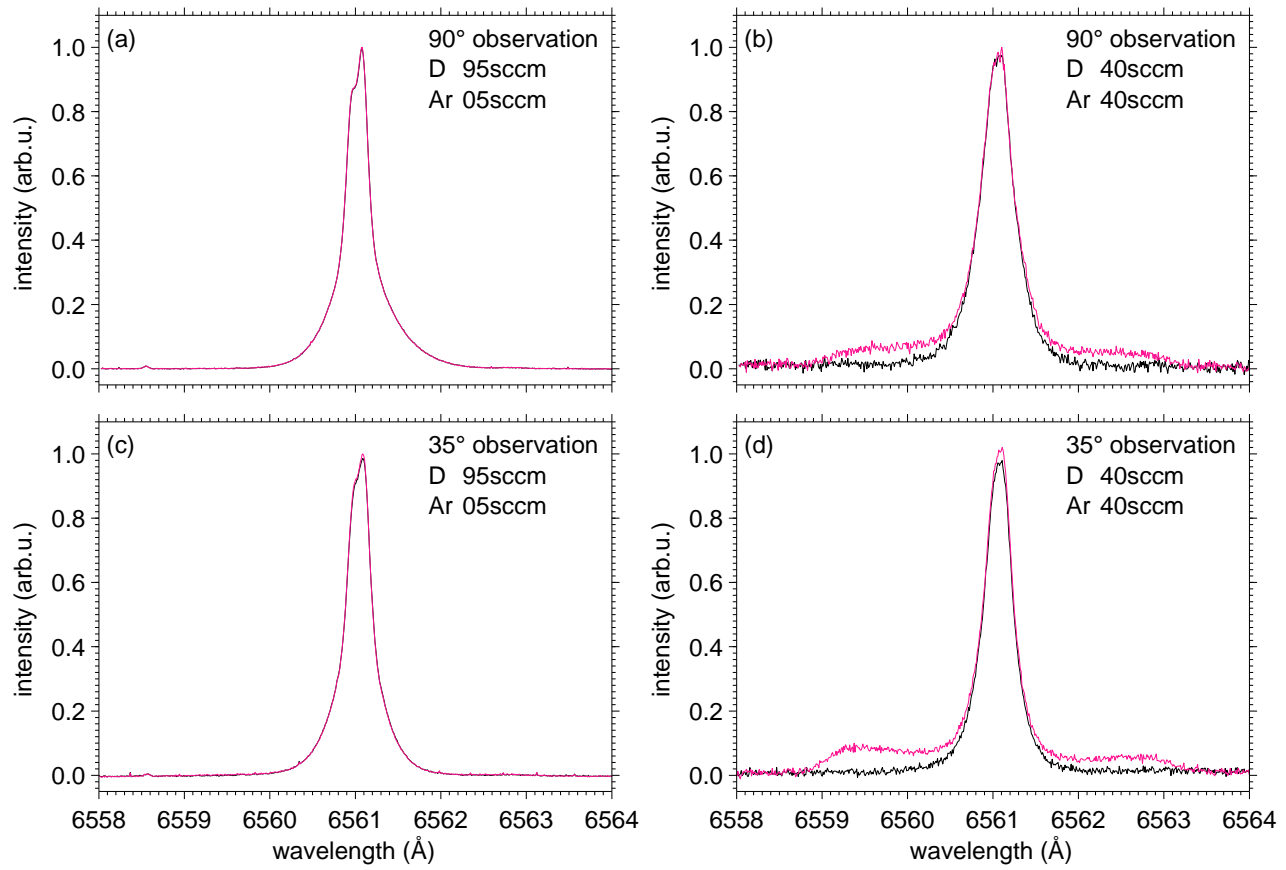

FIGURE 2. (Color online) $\mathrm{D}_{\alpha}$ line emission in the linear magnetized plasma in front of the target electrode measured with the Echelle spectrometer for two different gas conditions, (a,c) deuterium dominated plasma and (b,d) Ar-D mixed plasma. The black line shows the emission with the target electrode at floating potential $\left(V_{f}=-36 \mathrm{~V}\right)$ and the pink line the emission with an applied potential of $-110 \mathrm{~V}$ and $-120 \mathrm{~V}$. In $(\mathrm{a}, \mathrm{c})$ the black line is mostly hidden behind the pink line. In all cases gas pressure is $0.1 \mathrm{~Pa}$ and discharge current is 100 A. Figures (a,b) depict the observation at $90^{\circ}$ and (c,d) at $35^{\circ}$ relative to the target surface normal. In case of deuterium dominated plasma the D flow is $95 \mathrm{sccm}$ and argon flow is $5 \mathrm{sccm}$, in case of mixed plasma the flow of both Ar and D is $40 \mathrm{sccm}$. 
emission lines. Slightly different profiles of the $D_{\alpha}$ line in $(\mathrm{a}, \mathrm{c})$ are attributed to the projection of poloidal rotation velocity of the ions on different lines-of-sight [13]. The measured profiles of $D_{\alpha}$ and $D_{\beta}$ lines are used to derive the ion temperature of the plasma. An absolutely different picture appears in Ar-D plasma. By mixing hydrogen with argon in practically equal proportion, strong emission of fast atoms is observed at both lines-of-sight by applying a negative potential (here $-120 \mathrm{~V}$ ) to the target as shown in Figs. 2(b,d). (The variation of the maximal Doppler-shifted components in Ar-D plasma with the electrostatic potential at the target electrode is shown in details in Fig. 3 in Ref. [14].) The $90^{\circ}$ line-of-sight observes the plasma just in front of the target surface [Fig. 2(b)]. In this case the atoms moving toward the observer emit blue-shifted photons and the atoms moving away from the observer emit red-shifted photons. The detected photons appear in broad wings of symmetrical radiation around the unshifted $\mathrm{D}_{\alpha}$ line. The fast atoms moving perpendicular to the target, namely along the $z$ axis, emit the photons observed at the positions of the unshifted $\mathrm{D}_{\alpha}$ line, increasing its intensity only. The radiation caused by fast atoms increases the total integral emission of the $\mathrm{D}_{\alpha}$ line up to $30 \%$ for this line-of-sight. The emission detected at the angle of $35^{\circ}$ in Fig. 2(d) indicates also redand blue-shifted wings, however, here the emission profile is asymmetric. The reason for the red-shifted component, as discussed in Refs. [3, 4] is a result of photon reflection at the wall. In general the emission of photons can be assumed to be isotropic and the photons emitted by neutral atoms could be reflected by the target surface and reach the front optics as well [cf. Fig. 3 in Ref. [14]]. The reflectance for a tungsten surface at the wavelength of $\mathrm{D}_{\alpha}$ line equals to 0.504 [15]. The derived ratio between the intensity of the red- and blue-shifted emission is found to be $0.55 \pm 0.05$, being in rather good agreement with the theoretical value. The observed emission profiles shown in Figs. 2(b,d) agree with general consideration, e.g., the maximal velocity gained by deuterium ions in the $\approx 120 \mathrm{~V}$ sheath potential could be as high as $1.07 \times 10^{5} \mathrm{~m} / \mathrm{s}$ and the fast atom emission should be displaced by $2.347 \AA$ relative to the unshifted wavelength. This shift is indeed observed in Figs. 2(b,d).

Figure 3 compares the Balmer- $\alpha$ lines measured in Ar-D and Ar-H mixed plasma at approximately equal plasma parameters and operation conditions. In both cases the applied potential at the target electrode has been $-140 \mathrm{~V}$ relative to the vacuum chamber potential $(0 \mathrm{~V})$. For both plasmas the contribution of emission from fast neutral atoms is observed as Doppler blue-shifted and Doppler red-shifted wings [Fig. 3(a,b)]. Without the applied negative potential no Doppler-shifted components are observed (black line). The width of the Doppler-shifted wings is larger for the $H_{\alpha}$ line, because the velocity of the $H^{+}$ions is given by $v_{H} \approx\left(2 E / m_{H}\right)^{1 / 2} \approx\left(4 E / m_{D}\right)^{1 / 2}=\sqrt{(2)} v_{D}$. In Fig. 3(c) both the $H_{\alpha}$ line and the $D_{\alpha}$ line are plotted versus the wavelength difference. The superimposed energy axis are calculated using the relation $E=m v^{2} / 2=m / 2\left\{c\left(\lambda-\lambda_{0}\right) / \lambda_{0}\right\}^{2}$, with $\lambda_{0}$ being the weighted center wavelength of the corresponding emission line. Figure 4 shows the Doppler-shifted components for the first three Balmer lines $\left(D_{\alpha}, D_{\beta}\right.$ and $\left.D_{\gamma}\right)$ as a
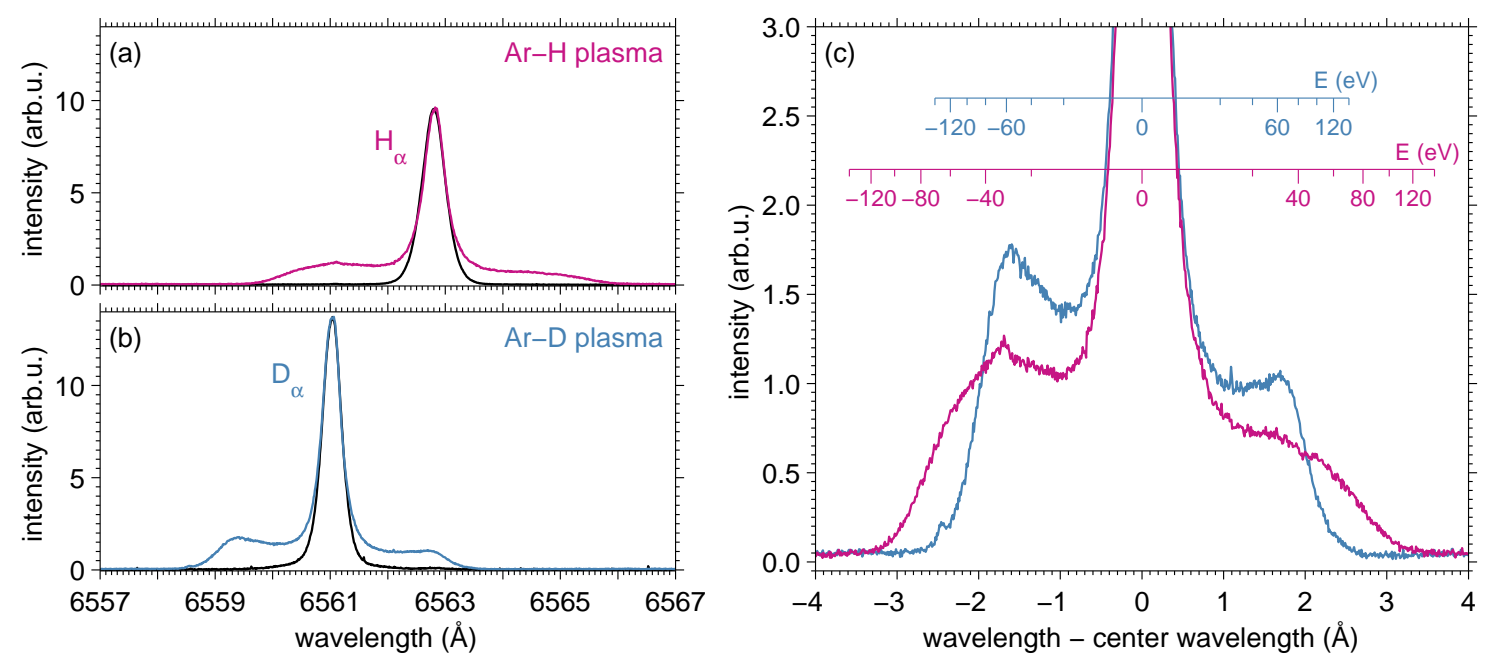

FIGURE 3. (Color online) The Balmer- $\alpha$ lines observed in (a) Ar-H and (b) Ar-D mixed plasma detected along the $35^{\circ}$ line-ofsight with the Echelle spectrometer. Argon gas flow into the source and hydrogen or deuterium gas flow into the plasma chamber have both been $80 \mathrm{sccm}$, floating potential has been at $-21 \mathrm{~V}$. In $(\mathrm{a}, \mathrm{b})$ the emission profiles for the cases with the target electrode being at floating potential is plotted in black color. In (c) both measurements are plotted versus the wavelength difference. The energy scales for hydrogen and deuterium are superimposed. 

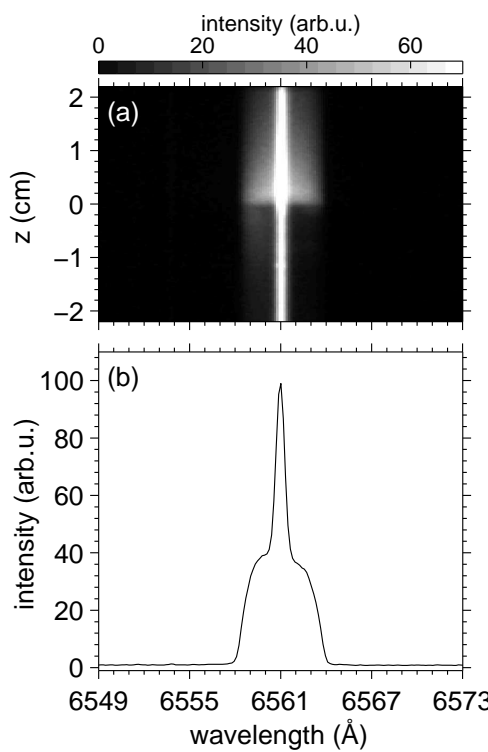
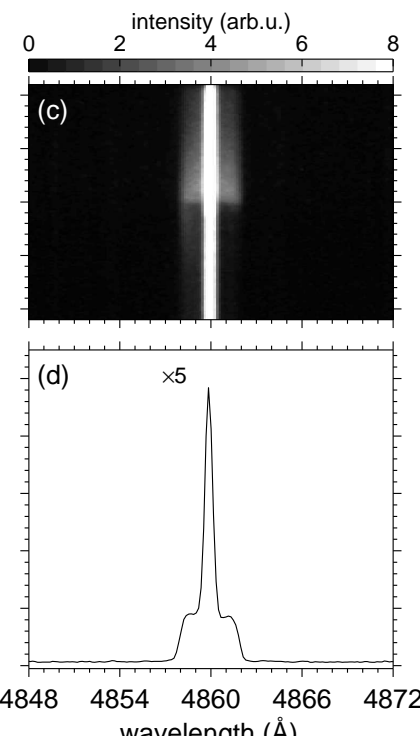
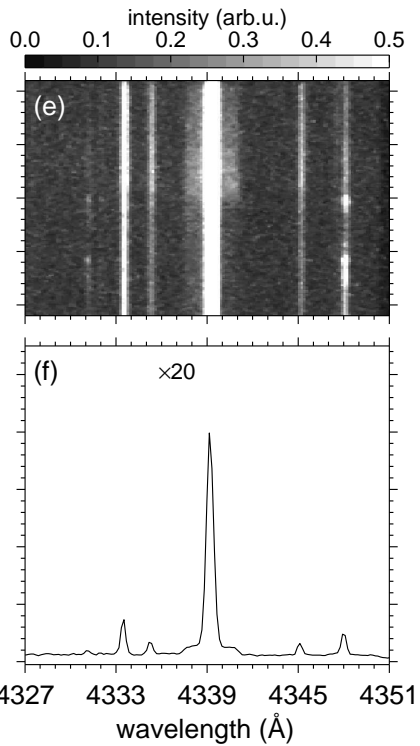

FIGURE 4. Emission of fast atoms observed from $90^{\circ}$ relative to the plasma axis in the (a,b) $\mathrm{D}_{\alpha},(\mathrm{c}, \mathrm{d}) \mathrm{D}_{\beta}$ and $(\mathrm{e}, \mathrm{f}) \mathrm{D}_{\gamma}$ line. The target electrode surface is at $z=0 \mathrm{~cm}$, the electrode potential is at $-200 \mathrm{~V}$ and the plasma volume is located at $z>0 \mathrm{~cm}$. The lower graphs show the averaged spectra over the first $5 \mathrm{~mm}$ in the plasma. The plasma operation parameters are Ar gas flow $80 \mathrm{sccm}$ and $\mathrm{D}$ gas flow $80 \mathrm{sccm}$ at the source gas inlet, the discharge current $140 \mathrm{~A}$ and gas pressure $0.054 \mathrm{~Pa}$. The electron temperature is $7 \mathrm{eV}$, ion temperature is $3 \mathrm{eV}$ and electron density is $1 \times 10^{18} \mathrm{~m}^{-3}$. In case of $D_{\gamma}$ line one also observes the lines of $\mathrm{Ar} \mathrm{I}$ at $4333.561 \AA$, $4335.338 \AA, 4345.168 \AA$ and the lines of Ar II at $4348.0635 \AA$ and $4331.1992 \AA$ (very weak).

function of distance from the target using the imaging spectrometer. First, we succeeded to observe the emission for all three components, though the intensity decreases dramatically going from the Balmer- $\alpha$ toward the Balmer- $\gamma$ line. Second, the emission of fast atoms is observed on the distances of a few $\mathrm{cm}$ in the plasma and was limited only by optical access to the plasma.

Similar to the measurements in Ar-H or Ar-D mixed plasmas we tried to observe fast atoms also in other gas mixtures. Figure 5 summarizes a series of measurements of deuterium mixed with the noble gases helium, neon, argon, krypton and xenon using the imaging spectrometer. For each gas mixture the plasma operation parameters, such as the discharge current $I_{d i s}$ and neutral gas pressure $p$, have been adjusted to find a stable discharge regime. Afterwards, the gas flow rates have been changed in order to achieve the maximum emission of fast atoms. Without applied acceleration potential to the target electrode, no wings can be observed around the $\mathrm{D}_{\alpha}$ line for all gas mixtures. With applied acceleration potential fast atoms can be distinctly observed in the plasma volume in front of the target electrode for $z>0 \mathrm{~cm}$ in Ar-D and Kr-D mixed plasmas, weak emission is also visible in Ne-D mixed plasma. (Fast atom emission observed for $z<0 \mathrm{~cm}$ results from reflected hydrogen atoms at the mounting of the target manipulator [compare Fig. 1].) From the same series of measurements Fig. 6(a) shows the $\mathrm{D}_{\alpha}$ line emission measured with the Echelle spectrometer from $35^{\circ}$ observation angle. Though the neutral gas pressure differs by a factor of two for different gas mixtures, one can conclude from Fig. 5 and Fig. 6(a) that the Ar-D plasma shows the strongest emission of fast atoms compared to the corresponding cold $\mathrm{D}_{\alpha}$ component among all mixed plasmas. In case of other gas mixtures the fast atoms emission is either rather dim (Ne-D) or could be hardly detected at all (He-D or Xe-D).

\section{DISCUSSION AND CONCLUSIONS}

The obtained experimental data allow following conclusions. First, the emission of fast atoms is the result of binary collisions between the atoms or ions of noble gases and fast hydrogen atoms reflected at the target electrode surface. The background hydrogen ions, atoms and molecules can not play the leading role in the excitation as the emission is missing in pure hydrogen or deuterium plasmas [cf. Fig. 2]. Second, the ions of noble gases could be also excluded as a source of excitation as follows from the theoretical data: the cross section of excitation of $\mathrm{H}$ atoms or $\mathrm{H}_{2}$ molecules 
target at floating potential


target with applied acceleration potential
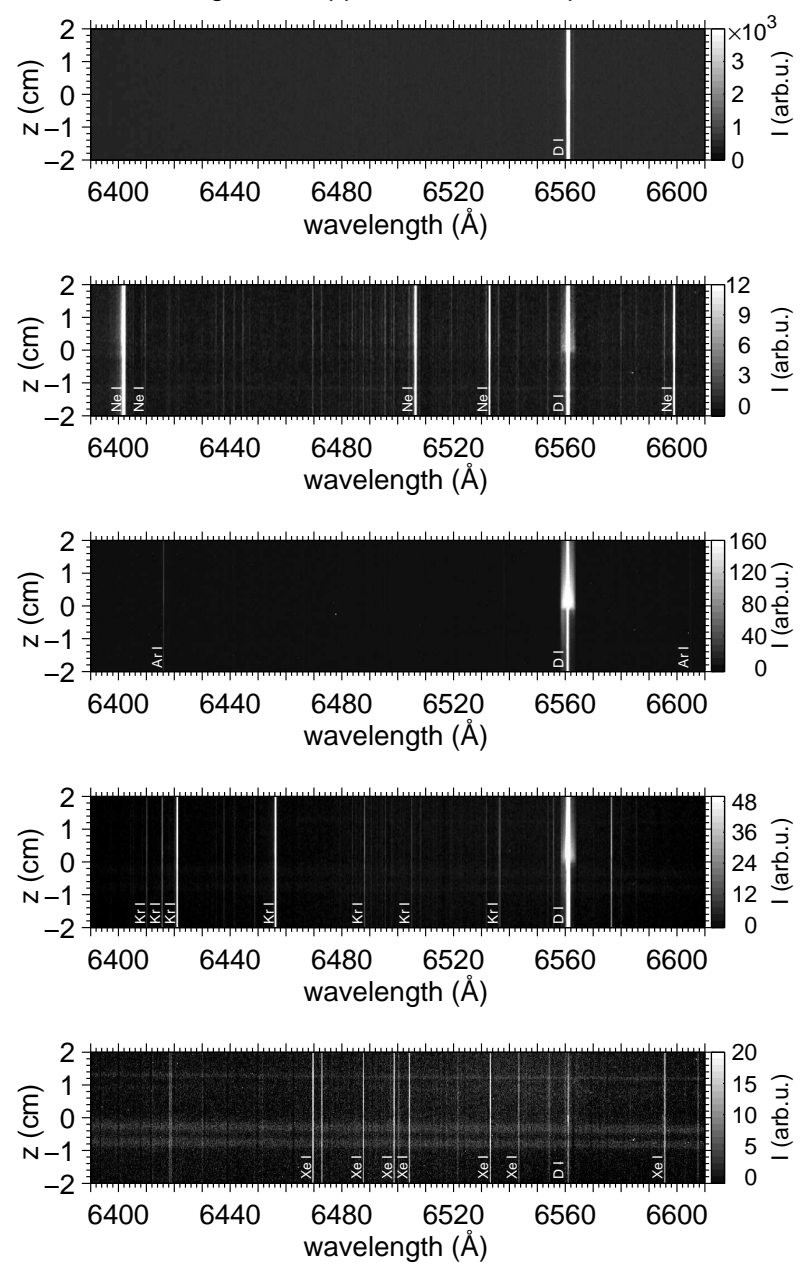

FIGURE 5. $\mathrm{D}_{\alpha}$ line emission measured with the imaging spectrometer for mixed plasmas of (a) He-D, (b) Ne-D, (c) Ar-D, (d) $\mathrm{Kr}-\mathrm{D}$ and (e) Xe-D. Figures on the left show the spectra for the target electrode at floating potential conditions. Figures on the right show the spectra in case of applied potential to the target (for He-D $-140 \mathrm{~V}$, for all others $-200 \mathrm{~V}$ ). The plotted light intensity $I$ is normalized to the integration time $\tau_{i}$. For the different mixed plasmas the operation parameters $\left(p, I_{d i s}\right.$, gas flows in sccm $\left.\Gamma_{D}: \Gamma_{\text {noble gas }}, \tau_{i}\right)$ have been as the following: (He-D) 0.033 Pa, $100 \mathrm{~A}, 20: 180,1 \mathrm{~s}$, (Ne-D) 0.039 Pa, $140 \mathrm{~A}, 80: 250,120 \mathrm{~s}$; (Ar-D) $0.054 \mathrm{~Pa}, 140 \mathrm{~A}, 80: 80,120 \mathrm{~s}$; (Kr-D) 0.034 Pa, 150 A, 70:30, $300 \mathrm{~s}$; (Xe-D) 0.075 Pa, 230 A, 70:30, $300 \mathrm{~s}$. The $z$ coordinate is organized as in Fig. 4.

by $\mathrm{Ar}^{+}$is 2-3 orders of magnitude lower compared to the excitation by $\mathrm{Ar}$ atoms [16]. So, for instance, the excitation cross section of the $\mathrm{H}_{\alpha}$ line by collision of the $\mathrm{H}_{2}$ molecule with $\mathrm{Ar}^{+}$is $1.3 \times 10^{-20} \mathrm{~cm}^{2}$ at the energy of $237 \mathrm{eV}$, whereas the excitation cross section by collisions of $\mathrm{H}$ atoms with Ar atoms equals to $0.65 \times 10^{-16} \mathrm{~cm}^{2}$ at the energy of $100 \mathrm{eV}$. The ionization balance for $\mathrm{Ar}$ or $\mathrm{Kr}$ was not measured in PSI-2, however, the ionic lines of $\mathrm{Ar}$ or $\mathrm{Kr}$ are rather weak in the spectra compared to Ar I lines (for instance see Fig. 4(e,f)). Here, the measured intensity of the strong Ar II line at $4348.1 \AA$ is comparable with the intensity of the weak lines of Ar I at $4333.6 \AA$ and $4335.3 \AA$ [17], indicating much lower densities of ion species in comparison with atomic argon. All these facts prove the leading role of neutral atom collisions leading to emission of spectral lines in the vicinity of the target:

$$
H_{f}+R g \rightarrow H_{f}^{*}+R g
$$

Here, $\mathrm{Rg}$ is the ground state of the noble gas ( $\mathrm{He}, \mathrm{Ne}, \mathrm{Ar}, \mathrm{Kr}$ or $\mathrm{Xe}$ ) and $\mathrm{H}_{f}$ and $\mathrm{H}_{f}^{*}$ are the atoms of hydrogen or deuterium in the ground or excited states, respectively. However, the major implication in the interpretation of the 

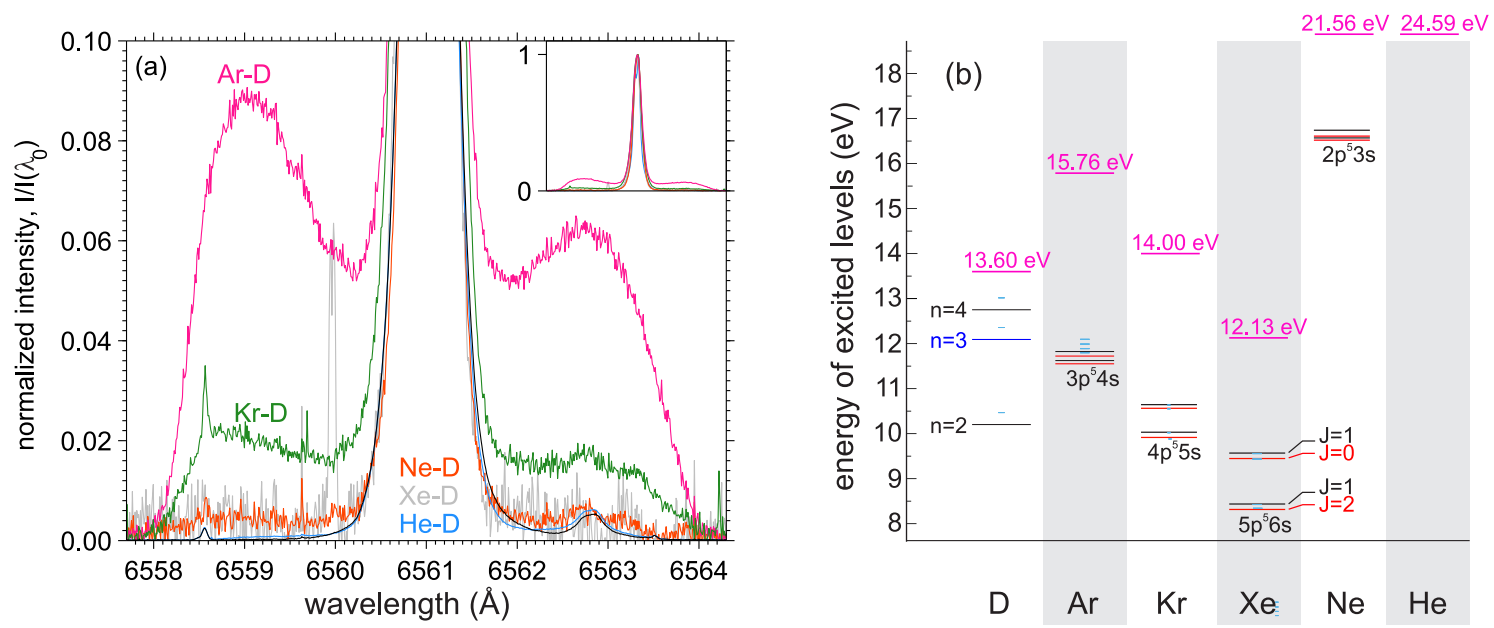

FIGURE 6. (Color online) (a) Measurement of fast atom emission from $35^{\circ}$ observation using the Echelle spectrometer for the same series of gas mixtures presented in Fig. 5. The black line corresponds to the measurement of He-D mixed plasma with the target being at floating potential. (b) Grotrian diagram of the first excited states of deuterium, argon, krypton, xenon, neon and helium [17]. The first ionization energy is depicted in magenta color. (The first excited states of He are out of range of the graph as well as the ionization energies of $\mathrm{He}$ and $\mathrm{Ne}$, which are shown for completeness only).

data appears by comparison of excitation cross sections of the $\mathrm{H}$ atom by collisions with noble gas atoms [18, 19, 20]. The excitation cross sections of the $\mathrm{H}$ atom by collisions with $\mathrm{Ar}$ and $\mathrm{Kr}$ atoms differ no more than $20 \%$ in the energy range of $80-200 \mathrm{eV}$ and the excitation cross sections by other noble gases are mainly one order of magnitude lower compared to Ar. The experimental data for fast atoms emission in mixed plasmas of He-D, Ne-D and Xe-D are in qualitative agreement with the theoretical cross sections. However, the experimental data in case of Ar-D mixed plasma shows stronger emission compared to the case of Kr-D mixed plasmas. This difference can not be explained by different gas flow ratios. Also the ionization rate coefficients of Ar and $\mathrm{Kr}$ differ no more than by a factor of two in the temperature range above $5 \mathrm{eV}$ [21], so that one does not expect a considerable attenuation of $\mathrm{Kr}$ compared to $\mathrm{Ar}$ at the densities of $10^{17}-10^{18} \mathrm{~m}^{-3}$. The difficulty in interpretation of the experimental data could be potentially resolved by looking for another source of excitation, namely the reaction of the so-called excitation transfer between the metastable states of $\mathrm{Ar}^{m}$ atoms and the ground state of $\mathrm{H}$ or $\mathrm{D}$ atoms:

$$
H_{f}+A r^{m} \rightarrow A r H^{*} \rightarrow H_{f}^{*}+A r .
$$

Here, $\mathrm{Ar}^{m}$ is the metastable state of argon and $\mathrm{ArH}^{*}$ is the excited state of the dimer molecule being the intermediate state of the reaction [22]. Indeed, in our plasmas, one expects a high concentration of metastable states relative to the ground state. Collisional quenching of the metastable fraction by collisions is rather low since low density and strong diffusion of metastables is expected.

In general, reaction (2) can potentially explain the anomalous strong emission of fast atoms in case of argon relative to all other noble gases. Indeed, the population of metastable fraction is proportional to the concentration of Ar atoms so that the emission behaves quite similar to the excitation by ground state. Second, reaction (1) and reaction (2) create the dimer molecule of $\mathrm{ArH}$ as the intermediate state between input and output channels [19,22]. The reaction of energy transfer must be especially efficient in case of $\mathrm{Ar}$ and $\mathrm{H}$ atom collisions as the metastable energy levels and excited states of atomic hydrogen are still relatively close to each other $(\Delta E=-0.539 \mathrm{eV}$ and $\Delta E=-0.364 \mathrm{eV}$ between two metastable states of Ar and the $n=3$ state of atomic hydrogen) [cf. Fig. 6(b)]. For excitation of $n=2$ levels of hydrogen the reaction is exothermic $(\Delta E=1.34955 \mathrm{eV}$ and $\Delta E=1.5243 \mathrm{eV}$ between the same metastable states of $\operatorname{Ar}$ and $n=2$ ), e.g., no kinetic energy is required to excite the atoms. The latter case was intensively studied for the $L_{\alpha}$ excitation and represents an extremely strong source of lines emission in afterglow discharges, though it was never studied in the energy range of $60-100 \mathrm{eV}$. The theoretical data for the $L_{\alpha}$ cross sections were found ten times higher as observed in the experiment and these results remain until now unexplained [23]. The excitation cross sections in case of endothermic reactions, which is the case for all other excited states of hydrogen, are according to the knowledge of the authors not available in literature, though considerable efforts were undertaken to calculate the 
potential curves of the ArH molecule [24]. We hope that our experimental findings stimulate the calculation of cross sections for Ar-H or Ar-D atomic systems. In case of other gases, such as $\mathrm{He}$ or $\mathrm{Ne}$ the reaction of excitation transfer (2) is not possible and for $\mathrm{Kr}$ and Xe the metastable states are energetically much lower compared to $n=3$ or $n=4$ levels of $\mathrm{H}$ atom to stimulate a considerable increase of emission. Independent on theoretical calculations a dedicated experimental setup of optical components is foreseen at PSI-2 to resolve this problem. So, for instance, one expects to achieve the spatial resolution using the setup of the imaging spectrometer on the order of few $\mu \mathrm{m}$ in direction perpendicular to the target electrode surface. We hope to obtain the temporal evolution of the emission profile in front of the target electrode to conclude on the source of the emission. In case of atoms moving away from the target the rise of the emission must be expected on the timescale of lifetime of excited states of $n=3,4,5$. Therefore a tunable diode laser system [25] is going to be installed at PSI-2 to measure the metastable fraction of Ar close to the target. If reaction (2) is the source of the emission, a difference of the population of metastable argon is expected, when comparing the emission without and with applied acceleration potential. With applied negative potential the metastable fraction of argon must decrease resulting in reduction of the absorbed laser intensity. Using different target electrode materials could give new insights on the energy distribution function of fast atoms and exclude the impact charge-exchange process of accelerated $\mathrm{H}^{+}$ions towards the target with noble gas atoms.

\section{ACKNOWLEDGMENTS}

We thank the PSI-2 Team for valuable discussion and technical support.

\section{REFERENCES}

[1] J. T. Clarke, J. Trauger, and J. H. Waite, Geophys. Res. Lett. 16, 587-590 (1989).

[2] J. M. Ajello, I. Kanik, and S. Ahmed, J. Geophys. Res. 100, 26411-26420 (1995).

[3] N. M. Sišović, G. L. Majstorović, and N. Konjević, Eur. Phys. J. D 32, 347-354 (2005).

[4] T. Babkina, T. Gans, and U. Czarnetzki, Europhys. Lett. 72, 235-241 (2005).

[5] C. Oliveira, J. A. Souza Corrêa, M. P. Gomes, B. N. Sismanoglu, and J. Amorim, Appl. Phys. Lett. 93, p. 041503 (2008).

[6] P. C. Stangeby, The Plasma Boundary of Magnetic Fusion Devices (Institute of Physics Publishing (London), 2000).

[7] W. Eckstein, Computer Simulation of Ion-Solid Interactions (Springer Series in Material Science, 1991).

[8] J. F. Ziegler, "SRIM/TRIM code," in www.srim.org (accessed May 2016).

[9] A. Kreter, C. Brandt, A. Huber, S. Kraus, S. Möller, M. Reinhart, B. Schweer, G. Sergienko, and B. Unterberg, Fusion Sci. Technol. 68, 8-14 (2015).

[10] http://www.andor.com/scientific-cameras/ixon-emccd-camera series, (2016).

[11] ftp://ftp.princetoninstruments.com/public/Manuals/Acton/SP 2750.pdf, "Operating Instructions, Acton Series SP-2750," (2016).

[12] http://www.andor.com/scientific-cameras/newton-ccd-and-emccd cameras, (2016).

[13] O. Waldmann, H. Meyer, and G. Fussmann, Contrib. Plasma. Phys. 47, 691-702 (2007).

[14] C. Brandt, O. Marchuk, A. Pospieszczyk, S. Brezinsek, M. Reinhart, and B. Unterberg, "Fast nonmaxwellian atoms in a linear magnetized plasma," in 42nd EPS Conference on Plasma Physics, O3.J107 (2015).

[15] M. N. Polyanskiy, "Refractive index database," in http://refractiveindex.info (accessed May 2016).

[16] A. V. Phelps, J. Phys. Chem. Ref. Data 21(4), 883-897 (1992).

[17] A. Kramida, Yu. Ralchenko, J. Reader, and and NIST ASD Team, NIST Atomic Spectra Database (ver. 5.3), [Online]. Available: http://physics.nist.gov/asd [2016, August 29]. National Institute of Standards and Technology, Gaithersburg, MD. (2015).

[18] B. Van Zyl, H. Neumann, H. L. Rothwell, Jr., and R. C. Amme, Phys. Rev. A 21(3), 716-729 (1980).

[19] B. Van Zyl, M. W. Gealy, and H. Neumann, Phys. Rev. A 31(5), 2922-2931 (1985).

[20] B. Van Zyl, H. Neumann, and M. W. Gealy, Phys. Rev. A 33(3), 2093-2095 (1986).

[21] S. Theiss, N. Bibinov, N. Bagcivan, M. Ewering, P. Awakowicz, and K. Bobzin, J. Phys. D: Appl. Phys 43, p. 075205 (2010).

[22] M. A. Clyne, M. C. Heaven, K. D. Bayes, and P. B. Monkhouse, Chemical Physics 47, 179-188 (1980).

[23] R. L. Vance and G. A. Gallup, J. Chem. Phys. 73(2), 894-901 (1980).

[24] A. Kirrander, M. S. Child, and A. V. Stolyarov, Phys. Chem. Chem. Phys. 8(2), 247-255 (2006).

[25] B. Du, "Plasma dynamics in the micro thin-cathode discharge," dissertation, Ruhr-University Bochum 2012.

[26] W. Allison, J. W. Sheldon, and E. E. Muschlitz, J. Phys. B: At. Mol. Phys. 14, 4587-4594 (1981). 